\title{
Multifractional Poisson process, multistable subordinator and related limit theorems
}

\author{
Ilya Molchanov ${ }^{\mathrm{a}}$, Kostiantyn Ralchenko ${ }^{\mathrm{a}, \mathrm{b}, *}$ \\ ${ }^{a}$ University of Bern, Institute of Mathematical Statistics and Actuarial Science, \\ Sidlerstrasse 5, CH-3012 Bern, Switzerland \\ ${ }^{b}$ Taras Shevchenko National University of Kyiv, Department of Probability Theory, \\ Statistics and Actuarial Mathematics, Volodymyrska 64/13, 01601 Kyiv, Ukraine
}

\begin{abstract}
We introduce a multistable subordinator, which generalizes the stable subordinator to the case of time-varying stability index. This enables us to define a multifractional Poisson process. We study properties of these processes and establish the convergence of a continuous-time random walk to the multifractional Poisson process.
\end{abstract}

Keywords: multistable process, multifractional process, stable subordinator, fractional Poisson process, continuous-time random walk

2010 MSC: 60G52, 60G22

\section{Introduction}

The multistable processes are generalizations of well-known stable processes. They are locally stable, but the stability index may evolve over time. Various definitions of such processes can be found in Falconer and Liu (2012); Falconer et al. (2009); Falconer and Lévy Véhel (2009); Le Guével and Lévy Véhel (2012); Le Guével et al. (2013).

The literature contains several definitions of the fractional Poisson process, see Beghin and Orsingher (2009); Laskin (2003); Mainardi et al. (2004); Repin and Saichev (2000); Uchaikin et al. (2008). Meerschaert et al. (2011) suggested to define a fractional Poisson process as $N(E(t))$, for a Poisson process $N(t)$ and $E(t)$ being the right-continuous inverse of the standard $\beta$-stable subordinator $D(t)$, independent of $N(t)$.

In this paper, we construct a multifractional Poisson process using the multistable subordinator instead of $D(t)$. This process has non-stationary increments, but it retains some important properties of the usual $\beta$-stable subordinator such as the stochastic continuity, the independence of the increments and the strictly

\footnotetext{
${ }^{*}$ Corresponding author

Email addresses: ilya.molchanov@stat.unibe.ch (Ilya Molchanov), k.ralchenko@gmail.com (Kostiantyn Ralchenko)
} 
increasing sample paths. We establish that the multistable subordinator is a weak limit of the sums $\sum_{k \leq n t} b_{n k}^{-1} J_{n k}$ where $\left\{J_{n k}, n \geq 1, k \geq 1\right\}$ are independent random variables with regularly varying tails and $\left\{b_{n k}, n \geq 1, k \geq 1\right\}$ are some normalizing constants. Moreover, we prove that the corresponding rightcontinuous inverse converges to the right-continuous inverse of the multistable subordinator. This makes possible to construct continuous-time random walks, which converge to the multifractional Poisson process. These results generalize (Meerschaert et al., 2011, Theorem 2.5) and (Meerschaert and Scheffler, 2004, Theorem 3.2) to the multistable case.

The paper is organized as follows. In Section 2 we introduce multistable subordinator, its inverse and multifractional Poisson process, and investigate their properties. In Section 3 we prove various limit theorems for these processes.

\section{Multistable subordinator and multifractional Poisson process}

\subsection{Basic definitions}

Let $\beta: \mathbb{R}_{+} \rightarrow(0,1)$ be a continuous function, $\mathbb{R}_{+}=[0, \infty)$. Denote

$$
\beta^{*}=\sup _{t} \beta(t), \quad \beta_{*}=\inf _{t} \beta(t) .
$$

Consider a Poisson point process $\Pi=\left\{\left(t_{i}, x_{i}\right)\right\}$ on $\mathbb{R}_{+} \times(0, \infty)$ with intensity measure

$$
\nu(d t, d x)=\beta(t) x^{-\beta(t)-1} d t d x .
$$

Define

$$
D(t)=\sum_{\substack{t_{i} \leq t \\\left(t_{i}, x_{i}\right) \in \Pi}} x_{i}, \quad t \geq 0
$$

Since

$$
\int_{[0, t] \times(0, \infty)} \min (1, x) \nu(d s, d x)=\int_{0}^{t} \frac{d s}{1-\beta(s)},
$$

the process $D(t)$ is well defined, if the function $(1-\beta(s))^{-1}$ is integrable. In particular, this condition holds if $\beta^{*}<1$. It follows from Fisz and Varadarajan (1962/1963) that the distribution of $D(t)$ is absolutely continuous for all $t>0$.

The Laplace transform of $D(t)$ is equal to

$$
\begin{aligned}
\mathrm{E} \exp \{-\theta D(t)\} & =\exp \left\{\int_{0}^{t} \int_{0}^{\infty}\left(e^{-\theta x}-1\right) \beta(s) x^{-\beta(s)-1} d x d s\right\} \\
& =\exp \left\{-\int_{0}^{t} \Gamma(1-\beta(s)) \theta^{\beta(s)} d s\right\}
\end{aligned}
$$

see, for instance, (Kingman, 1993, Section 3.2). If $\beta(t)=\beta$ is a constant,

$$
\mathrm{E} \exp \{-\theta D(t)\}=\exp \left\{-\Gamma(1-\beta) t \theta^{\beta}\right\},
$$

is the Laplace transform of the $\beta$-stable subordinator. We will call the process $D(t)$ the multistable subordinator with index $\beta(t), t \geq 0$. 
Remark 2.1. It is not hard to see that $D(t)$ can be represented as a sum over the stationary Poisson point process $\Pi^{\prime}$ on $\mathbb{R}_{+} \times(0, \infty)$ (with the Lebesgue intensity measure), namely

$$
D(t) \stackrel{d}{=} \sum_{\substack{t_{i} \leq t \\\left(t_{i}, x_{i}\right) \in \Pi^{\prime}}} x_{i}^{-1 / \beta\left(t_{i}\right)} .
$$

Remark 2.2. Le Guével et al. (2013) study the so-called independent increments multistable Lévy motion. It admits the following representation (Le Guével et al., 2013, Proposition 1):

$$
L(t)=\sum_{\substack{t_{i} \leq t \\\left(t_{i}, x_{i}\right) \in \Pi^{\prime \prime}}} C_{\beta\left(t_{i}\right)}^{1 / \beta\left(t_{i}\right)} x_{i}^{<-1 / \beta\left(t_{i}\right)>}, \quad t \in \mathbb{R}_{+},
$$

where $\Pi^{\prime \prime}$ is a stationary Poisson point process on $\mathbb{R}_{+} \times \mathbb{R}, C_{\beta}=\Gamma(1-\beta) \cos \frac{\beta \pi}{2}$ and $x_{i}^{<-1 / \beta\left(t_{i}\right)>}=\operatorname{sign}\left(x_{i}\right)\left|x_{i}\right|^{-1 / \beta\left(t_{i}\right)}$. We construct a multistable process using a similar approach, but we avoid using factor $C_{\beta}$ and consider only positive $x$ 's in order to obtain a process with positive and strictly increasing sample paths. Note that the sample paths of $L(t)$ are piecewise-constant functions, see (Le Guével et al., 2013).

Let $E(r)$ be the right-continuous inverse of $D(t)$, i. e.

$$
E(r)=\inf \{t \geq 0: D(t) \geq r\} .
$$

Furthermore, let $N(t)$ be a homogeneous Poisson process with intensity $\lambda$, independent of $\Pi$.

Definition 2.3. We call the process $X(t)=N(E(t)), t \geq 0$, multifractional Poisson process with index $\beta(t), t \geq 0$.

\subsection{Properties of the multistable subordinator}

In this subsection we will prove some useful properties of the processes $D(t)$ and $E(t)$.

Proposition 2.4. Assume that $\beta^{*}<1$. Then the process $D$ is continuous in probability. Moreover, for any $\varepsilon>0$ there exists a constant $C_{\varepsilon}>0$ such that

$$
\mathrm{P}(D(t+h)-D(t)>\varepsilon) \leq C_{\varepsilon} h
$$

for all $t, h \in \mathbb{R}_{+}$.

Proof. We have

$$
\begin{aligned}
\mathrm{P}(D(t+h)- & D(t)>\varepsilon) \\
& \leq \mathrm{P}\left(\sum_{\substack{t<t_{i} \leq t+h, x_{i} \leq 1 \\
\left(t_{i}, x_{i}\right) \in \Pi}} x_{i}>\frac{\varepsilon}{2}\right)+\mathrm{P}\left(\sum_{\substack{t<t_{i} \leq t+h, x_{i}>1 \\
\left(t_{i}, x_{i}\right) \in \Pi}} x_{i}>\frac{\varepsilon}{2}\right) .
\end{aligned}
$$


By Markov's inequality, we obtain

$$
\begin{gathered}
\mathrm{P}\left(\sum_{\substack{t<t_{i} \leq t+h, x_{i} \leq 1 \\
\left(t_{i}, x_{i}\right) \in \Pi}} x_{i}>\frac{\varepsilon}{2}\right) \leq \frac{2}{\varepsilon} \mathrm{E} \sum_{\substack{t<t_{i} \leq t+h, x_{i} \leq 1 \\
\left(t_{i}, x_{i}\right) \in \Pi}} x_{i} \\
=\frac{2}{\varepsilon} \int_{t}^{t+h} \int_{0}^{1} \beta(s) x^{-\beta(s)} d x d s=\frac{2}{\varepsilon} \int_{t}^{t+h} \frac{\beta(s)}{1-\beta(s)} d s \leq \frac{2 \beta^{*}}{\varepsilon\left(1-\beta^{*}\right)} h .
\end{gathered}
$$

The inequality under the ultimate probability in (4) implies that the Poisson process $\Pi$ has at least one point in $(t, t+h] \times(1, \infty)$. Therefore,

$$
\begin{array}{r}
\mathrm{P}\left(\sum_{\substack{t<t_{i} \leq t+h, x_{i}>1 \\
\left(t_{i}, x_{i}\right) \in \Pi}} x_{i}>\frac{\varepsilon}{2}\right) \\
\leq 1-\exp \left\{-\int_{t}^{t+h} \int_{1}^{\infty} \beta(s) x^{-\beta(s)-1} d s d x\right\}=1-e^{-h} \leq h .
\end{array}
$$

Thus, (3) holds with $C_{\varepsilon}=1+\frac{2 \beta^{*}}{\varepsilon\left(1-\beta^{*}\right)}$.

Proposition 2.5. The process $D(t), t \geq 0$ has independent increments.

Proof. The result follows from the Poisson property of $\Pi$.

Proposition 2.6. The sample paths of $D$ are a.s. strictly increasing.

Proof. For all $t \geq 0$ and $h>0$, the event $\{D(t+h)=D(t)\}$ means that the Poisson process $\Pi$ has no points in $(t, t+h] \times(0, \infty)$. Therefore, $\mathrm{P}(D(t+h)=$ $D(t))=0$. Then $D$ is strictly increasing by considering rational $t$ and $h$.

Corollary 2.7. The sample paths of $E$ are a.s. continuous and non-decreasing.

Proof. The statement follows directly from the definition of $E$ and Proposition 2.6.

\section{Limit theorems}

\subsection{Convergence of point processes in the scheme of series}

Let $\left\{J_{n k}, n \geq 1, k \geq 1\right\}$ be non-negative independent random variables such that

$$
\mathrm{P}\left(J_{n k}>t\right)=t^{-\beta(k / n)} L\left(t^{\beta(k / n) / \beta^{*}}\right)
$$

for some slowly varying function $L(t)$, i. e.

$$
L(\lambda t) / L(t) \rightarrow 1 \text { as } t \rightarrow \infty \text { for any } \lambda>0 .
$$


Define the sequences of normalizing constants $\left\{a_{n}, n \geq 1\right\}$ and $\left\{b_{n k}, n \geq 1, k \geq\right.$ $1\}$ by

$$
\begin{aligned}
& a_{n}^{-\beta^{*}} L\left(a_{n}\right)=\frac{1}{n}, \\
& b_{n k}=a_{n}^{\beta^{*} / \beta(k / n)} .
\end{aligned}
$$

Let $\delta_{x}$ be the delta-measure concentrated at $x$.

Theorem 3.1. The point process $N_{n}=\sum_{k=1}^{\infty} \delta_{\left(k n^{-1}, b_{n k}^{-1} J_{n k}\right)}$ weakly converges to the Poisson point process $\Pi$.

Proof. The proof follows the scheme from (Resnick, 1987, Proposition 3.21). According to (Resnick, 1987, Proposition 3.19), it suffices to prove the convergence of Laplace functionals $\psi_{N_{n}}(f) \rightarrow \psi_{\Pi}(f)$ for arbitrary function $f$ from the family $C_{0}^{+}\left(\mathbb{R}_{+} \times(0, \infty)\right)$ of continuous, non-negative functions on $\mathbb{R}_{+} \times(0, \infty)$ with compact support. We have

$$
\psi_{\Pi}(f)=\exp \left\{-\int_{\mathbb{R}_{+} \times(0, \infty)}\left(1-e^{-f(s, x)}\right) \nu(d s, d x)\right\},
$$

see, for example, (Resnick, 1987, Proposition 3.6), and

$$
\begin{aligned}
\psi_{N_{n}}(f) & =\mathrm{E} \exp \left\{-\sum_{k} f\left(k n^{-1}, b_{n k}^{-1} J_{n k}\right)\right\} \\
& =\prod_{k}\left(1-\int_{0}^{\infty}\left(1-e^{-f\left(k n^{-1}, b_{n k}^{-1} y\right)}\right) \mathrm{P}\left(J_{n k} \in d y\right)\right) \\
& =\prod_{k}\left(1-\int_{0}^{\infty}\left(1-e^{-f\left(k n^{-1}, x\right)}\right) \mathrm{P}\left(b_{n k}^{-1} J_{n k} \in d x\right)\right) .
\end{aligned}
$$

Consider the measure $\mu_{n k}(\cdot)=\mathrm{P}\left(b_{n k}^{-1} J_{n k} \in \cdot\right)$. We claim that

$$
\lim _{n \rightarrow \infty} \sup _{k}\left|n \mu_{n k}\left(\left[c_{1}, c_{2}\right]\right)-\left(c_{1}^{-\beta(k / n)}-c_{2}^{-\beta(k / n)}\right)\right|=0
$$

for any $\left[c_{1}, c_{2}\right] \subset(0, \infty)$. Indeed, using the relations (5), (7) and (8), we can write

$$
\begin{aligned}
n \mu_{n k}\left(\left[c_{1}, c_{2}\right]\right)= & n\left(\mathrm{P}\left(J_{n k}>b_{n k} c_{1}\right)-\mathrm{P}\left(J_{n k}>b_{n k} c_{2}\right)\right) \\
& =\frac{\left(b_{n k} c_{1}\right)^{-\beta(k / n)} L\left(\left(b_{n k} c_{1}\right)^{\frac{\beta(k / n)}{\beta^{*}}}\right)-\left(b_{n k} c_{2}\right)^{-\beta(k / n)} L\left(\left(b_{n k} c_{2}\right)^{\frac{\beta(k / n)}{\beta^{*}}}\right)}{a_{n}^{-\beta^{*}} L\left(a_{n}\right)} \\
& =c_{1}^{-\beta(k / n)} \frac{L\left(a_{n} c_{1}^{\frac{\beta(k / n)}{\beta^{*}}}\right)}{L\left(a_{n}\right)}-c_{2}^{-\beta(k / n)} \frac{L\left(a_{n} c_{2}^{\frac{\beta(k / n)}{\beta^{*}}}\right)}{L\left(a_{n}\right)} .
\end{aligned}
$$


It is known (see Seneta, 1976, Theorem 1.1) that for every fixed $\left[d_{1}, d_{2}\right] \subset(0, \infty)$ the convergence (6) holds uniformly with respect to $\lambda \in\left[d_{1}, d_{2}\right]$. Therefore, (10) follows from (11), since $a_{n} \rightarrow \infty$ as $n \rightarrow \infty$.

Define $\nu_{n}$ by

$$
\nu_{n}(d s, d x)=\sum_{k} \delta_{k n^{-1}}(d s) \mu_{n k}(d x) .
$$

Then

$$
\begin{aligned}
& \nu_{n}\left((u, v] \times\left(c_{1}, c_{2}\right]\right) \\
& \quad=\frac{1}{n} \sum_{k} \delta_{k n^{-1}}((u, v])\left(n \mu_{n k}\left(\left(c_{1}, c_{2}\right]\right)-\left(c_{1}^{-\beta\left(k n^{-1}\right)}-c_{2}^{-\beta\left(k n^{-1}\right)}\right)\right) \\
& \quad+\int_{c_{1}}^{c_{2}}\left(\frac{1}{n} \sum_{k} \delta_{k n^{-1}}((u, v]) \beta\left(k n^{-1}\right) x^{-\beta\left(k n^{-1}\right)-1}\right) d x
\end{aligned}
$$

The first term in the right-hand side converges to 0 as $n \rightarrow \infty$ by (10). The second one converges to $\nu\left((u, v] \times\left(c_{1}, c_{2}\right]\right)$, since the integrand is the Riemann sum for $\int_{u}^{v} \beta(s) x^{-\beta(s)-1} d s$. Hence, $\nu_{n}$ converges weakly to $\nu$.

Therefore,

$$
\begin{aligned}
\sum_{k} \int_{0}^{\infty}\left(1-e^{-f\left(k n^{-1}, x\right)}\right) \mu_{n k}(d x)=\int_{\mathbb{R}_{+} \times(0, \infty)}\left(1-e^{-f}\right) d \nu_{n} \\
\rightarrow \int_{\mathbb{R}_{+} \times(0, \infty)}\left(1-e^{-f(s, x)}\right) \nu(d s, d x)=-\log \psi_{\Pi}(f)
\end{aligned}
$$

as $n \rightarrow \infty$.

If $K \subset \mathbb{R}_{+} \times(0, \infty)$ is the compact support of $f$ and $\left[c_{1}, c_{2}\right] \subset(0, \infty)$ is its projection on the second coordinate, then

$$
\sup _{k} \int_{0}^{\infty}\left(1-e^{-f\left(k n^{-1}, x\right)}\right) \mu_{n k}(d x) \leq \sup _{k} \mu_{n k}\left(\left[c_{1}, c_{2}\right]\right) \rightarrow 0 \quad \text { as } n \rightarrow \infty
$$

by (10). Equation (9) implies

$$
-\log \psi_{N_{n}}(f)=-\sum_{k} \log \left(1-\int_{0}^{\infty}\left(1-e^{-f\left(k n^{-1}, x\right)}\right) \mu_{n k}(d x)\right) .
$$

The elementary expansion

$$
\log (1+z)=z(1+\varepsilon(z))
$$


for $|\varepsilon(z)| \leq|z|$ if $|z| \leq 1 / 2$ yields

$$
\begin{aligned}
& \mid-\log \psi_{N_{n}}(f)- \sum_{k} \int_{0}^{\infty}\left(1-e^{-f\left(k n^{-1}, x\right)}\right) \mu_{n k}(d x) \mid \\
& \leq \sum_{k}\left(\int_{0}^{\infty}\left(1-e^{-f\left(k n^{-1}, x\right)}\right) \mu_{n k}(d x)\right)^{2} \\
& \leq\left(\sup _{k} \int_{0}^{\infty}\left(1-e^{-f\left(k n^{-1}, x\right)}\right) \mu_{n k}(d x)\right) \\
& \quad \times \sum_{k} \int_{0}^{\infty}\left(1-e^{-f\left(k n^{-1}, x\right)}\right) \mu_{n k}(d x) \rightarrow 0
\end{aligned}
$$

as $n \rightarrow \infty$ by (12) and (13). Together with (12) this implies that

$$
-\log \psi_{N_{n}}(f) \rightarrow-\log \psi_{\Pi}(f) \quad \text { as } n \rightarrow \infty .
$$

Hence, $\psi_{N_{n}}(f) \rightarrow \psi_{\Pi}(f)$ as $n \rightarrow \infty$.

\subsection{Convergence of sums to the multistable subordinator}

Theorem 3.2. Assume that $\beta_{*}>0$ and $\beta^{*}<1$. Then $\sum_{k \leq n t} b_{n k}^{-1} J_{n k}$ weakly converges to $D(t)$ as $n \rightarrow \infty$.

Proof. By the Skorohod theorem, it is possible to define $N_{n}$ and $\Pi$ on the same probability space so that $N_{n} \rightarrow \Pi$ a.s as $n \rightarrow \infty$. For all $r \in(0,1)$ we have

$$
\begin{aligned}
\left|\sum_{k \leq n t} b_{n k}^{-1} J_{n k}-D(t)\right| \leq\left|\sum_{\substack{k \leq n t \\
b_{n k}^{-1} J_{n k} \leq r}} b_{n k}^{-1} J_{n k}\right| \\
+\left|\sum_{\substack{k \leq n t \\
b_{n k}^{-1} J_{n k} \geq r}} b_{n k}^{-1} J_{n k}-\sum_{\substack{t_{i} \leq t, x_{i} \geq r \\
\left(t_{i}, x_{i}\right) \in \Pi}} x_{i}\right|+\left|\sum_{\substack{t_{i} \leq t, x_{i} \leq r \\
\left(t_{i}, x_{i}\right) \in \Pi \\
\\
=\eta_{n}(r)+\zeta_{n}(r)+\gamma(r) .}} x_{i}\right|
\end{aligned}
$$

Note that the processes $N_{n}$ and $\Pi$ have a.s. at most finite number of points in $[0, t] \times[r, \infty)$ for every $t>0$ and $r>0$. Therefore the a.s. convergence $N_{n} \rightarrow \Pi$ implies that the second summand $\zeta_{n}(r) \rightarrow 0$ a.s. as $n \rightarrow \infty$ for all $r>0$.

The third summand $\gamma(r)=\int_{[0, t] \times(0, r]} x d \Pi \rightarrow 0$ a.s. as $r \downarrow 0$, since the integral $\int_{[0, t] \times(0,1]} x d \Pi$ is a.s. finite. Indeed, $\int_{[0, t] \times(0,1]} x \nu(d s, d x)<\infty$, see (11). 
Consider the first summand. If $r<1$, then

$$
\begin{aligned}
\eta_{n}(r) & =\sum_{\substack{k \leq n t \\
b_{n k}^{-1} J_{n k} \leq r}} b_{n k}^{-1} J_{n k} \leq \sum_{\substack{k \leq n t \\
b_{n k}^{-1} J_{n k} \leq r}}\left(b_{n k}^{-1} J_{n k}\right)^{\beta\left(k n^{-1}\right) / \beta^{*}} \\
& =a_{n}^{-1} \sum_{\substack{k \leq n t \\
b_{n k}^{-1} J_{n k} \leq r}} J_{n k}^{\beta\left(k n^{-1}\right) / \beta^{*}},
\end{aligned}
$$

where the last equality follows from (8). Similarly, we can rewrite the inequality $b_{n k}^{-1} J_{n k} \leq r$ in the following form

$$
a_{n}^{-1} J_{n k}^{\beta\left(k n^{-1}\right) / \beta^{*}} \leq r^{\beta\left(k n^{-1}\right) / \beta^{*}} .
$$

Then

$$
\eta_{n}(r) \leq \sum_{\substack{k \leq n t \\ a_{n}^{-1} J_{n k}^{\beta\left(k n^{-1}\right) / \beta^{*}} \leq r^{\beta * / \beta^{*}}}} a_{n}^{-1} J_{n k}^{\beta\left(k n^{-1}\right) / \beta^{*}}
$$

for any $r \in(0,1)$.

It follows from (5) that

$$
\mathrm{P}\left(J_{n k}^{\beta\left(k n^{-1}\right) / \beta^{*}}>t\right)=t^{-\beta^{*}} L(t) .
$$

This means that $\left\{J_{n k}^{\beta\left(k n^{-1}\right) / \beta^{*}}, n \geq 1, k \geq 1\right\}$ is a sequence of i. i. d. random variables with regularly varying tails. Binomial point processes generated by i.i.d. sequence of random variables with such tails were considered in Davydov et al. (2008) and the following property was established: if $\xi_{1}, \xi_{2}, \ldots$ are i.i.d. random variables with $\mathrm{P}\left(\xi_{1}>t\right)=t^{-\beta^{*}} L(t)$ and $\left\{a_{n}, n \geq 1\right\}$ is a corresponding sequence of normalizing constants, then the process $\beta_{n}=\sum_{k=1}^{n} \delta_{a_{n}^{-1} \xi_{k}}$ satisfies the condition

$$
\underset{n}{\limsup \mathrm{P}}\left(\left|\int_{[0, r]} x \beta_{n}(d x)\right| \geq \varepsilon\right) \rightarrow 0 \quad \text { as } r \downarrow 0
$$

for each $\varepsilon>0$ (see the proof of Theorem 4.6 in Davydov et al., 2008). Using this fact, we deduce from (14) that

$$
\lim \sup \mathrm{P}\left(\eta_{n}(r) \geq \varepsilon\right) \rightarrow 0 \quad \text { as } r \downarrow 0 .
$$

n

Thus,

$$
\begin{aligned}
\mathrm{P}\left(\left|\sum_{k \leq n t} b_{n k}^{-1} J_{n k}-D(t)\right| \geq \varepsilon\right) & \leq \mathrm{P}\left(\eta_{n}(r)+\zeta_{n}(r)+\gamma(r) \geq \varepsilon\right) \\
& \leq \mathrm{P}\left(\eta_{n}(r) \geq \varepsilon / 2\right)+\mathrm{P}\left(\zeta_{n}(r)+\gamma(r) \geq \varepsilon / 2\right) .
\end{aligned}
$$

This probability can be made arbitrary small by the choice of $n$ and $r$. 
Corollary 3.3. Assume that $\beta_{*}>0$ and $\beta^{*}<1$. Then the finite-dimensional distributions of $E_{n}(r)=\inf \left\{t: \sum_{k \leq n t} b_{n k}^{-1} J_{n k} \geq r\right\}$ converge to those of $E(r)$ as $n \rightarrow \infty$.

Proof. For arbitrary $m, r_{1}, \ldots, r_{m}$ and $u_{1}, \ldots, u_{m}$

$$
\begin{aligned}
& \mathrm{P}\left(\inf \left\{t: \sum_{k \leq n t} b_{n k}^{-1} J_{n k} \geq r_{j}\right\} \leq u_{j}, j=1, \ldots, m\right) \\
& \quad=\mathrm{P}\left(\sum_{k \leq n u_{j}} b_{n k}^{-1} J_{n k} \geq r_{j}, j=1, \ldots, m\right) \rightarrow \mathrm{P}\left(D\left(u_{j}\right) \geq r_{j}, j=1, \ldots, m\right) \\
& \quad=\mathrm{P}\left(\inf \left\{t: D(t) \geq r_{j}\right\} \leq u_{j}, j=1, \ldots, m\right) \\
& =\mathrm{P}\left(E\left(r_{j}\right) \leq u_{j}, j=1, \ldots, m\right)
\end{aligned}
$$

as $n \rightarrow \infty$ by Theorem 3.2 .

Corollary 3.4. Assume that $\beta_{*}>0$ and $\beta^{*}<1$. Then $E_{n}$ weakly converges to $E$ in $D\left(\mathbb{R}_{+}, \mathbb{R}_{+}\right)$as $n \rightarrow \infty$ in the $J_{1}$ topology.

Proof. Since $E_{n}$ has non-decreasing sample paths and $E$ is continuous in probability by Corollary 2.7, the convergence under $J_{1}$ follows from Corollary 3.3 and (Bingham, 1971, Theorem 3).

3.3. Convergence of continuous-time random walks to the multifractional Poisson process

Let $Y_{i}^{(p)}, i \geq 1$, be i. i. d. random variables, independent of $J_{n k}, n \geq 1, k \geq 1$, such that

$$
\mathrm{P}\left(Y_{i}^{(p)}=1\right)=p, \quad \mathrm{P}\left(Y_{i}^{(p)}=0\right)=1-p .
$$

Denote $S^{(p)}(t)=\sum_{i \leq t} Y_{i}^{(p)}$.

Theorem 3.5. Assume that $\beta_{*}>0$ and $\beta^{*}<1$. If $p_{n} \downarrow 0$ as $n \rightarrow \infty$, then the continuous-time random walk $S^{\left(p_{n}\right)}\left(\lambda E_{n}(t) / p_{n}\right)$ converges to the multifractional Poisson process $N(E(t))$ as $n \rightarrow \infty$ in the $M_{1}$ topology on $D\left(\mathbb{R}_{+}, \mathbb{R}\right)$.

Proof. It was established in the proof of (Meerschaert et al., 2011, Theorem $2.5)$ that $S^{(p)}(\lambda t / p)$ weakly converges to $N(t)$ as $p \rightarrow 0$ in the $J_{1}$ topology. By Corollary 3.4 and (Billingslev, 1968, Theorem 3.2), $\left(S^{\left(p_{n}\right)}\left(\lambda t / p_{n}\right), E_{n}(t)\right)$ weakly converges to $(N(t), E(t))$ as $n \rightarrow \infty$ in the $J_{1}$ topology of the product space $D\left(\mathbb{R}_{+}, \mathbb{R} \times \mathbb{R}\right)$. In order to prove the convergence of the compositions in the $M_{1}$ topology, we will apply (Whitt, 2002, Theorem 13.2.4). Taking into account the continuity of $E$ (Corollary 2.7), it is sufficient to check that $t=E(r)$ is (almost surely) strictly increasing at $r$ whenever $N(t-) \neq N(t)$. It is easy to see that this condition holds if and only if the processes $D$ and $N$ have (almost surely) no simultaneous jumps. Thus, we need to verify that the probability that both $D(t)-D(t-)$ and $N(t)-N(t-)$ exceed $\varepsilon$ for some $t \geq 0$ is equal to zero for 
arbitrary $\varepsilon>0$. Obviously, it is sufficient to prove this for a finite interval. For the sake of simplicity, consider the interval $[0,1]$. Then

$$
\begin{aligned}
& \mathrm{P}(D(t)-D(t-)>\varepsilon, N(t)-N(t-)>\varepsilon \text { for some } t \in[0,1]) \\
& \leq \mathrm{P}\left(\bigcap_{j=1}^{\infty} A_{2^{j}}\right)=\lim _{j \rightarrow \infty} \mathrm{P}\left(A_{2^{j}}\right),
\end{aligned}
$$

where $A_{j}=\bigcup_{k=0}^{j-1}\left\{D\left(\frac{k+1}{j}\right)-D\left(\frac{k}{j}\right)>\varepsilon, N\left(\frac{k+1}{j}\right)-N\left(\frac{k}{j}\right)>\varepsilon\right\}$. Using the independence of processes, the estimate (3) for $D$ and the stationarity of the increments for $N$, we obtain

$$
\begin{aligned}
\mathrm{P}\left(A_{j}\right) & \leq \sum_{k=0}^{j-1} \mathrm{P}\left(D\left(\frac{k+1}{j}\right)-D\left(\frac{k}{j}\right)>\varepsilon\right) \mathrm{P}\left(N\left(\frac{k+1}{j}\right)-N\left(\frac{k}{j}\right)>\varepsilon\right) \\
& \leq \frac{C_{\varepsilon}}{j} \sum_{k=0}^{j-1} \mathrm{P}\left(N\left(\frac{k+1}{j}\right)-N\left(\frac{k}{j}\right)>\varepsilon\right)=C_{\varepsilon} \mathrm{P}(N(1 / j)>\varepsilon) .
\end{aligned}
$$

Since the Poisson process $N$ is continuous in probability, we have $\mathrm{P}\left(A_{j}\right) \rightarrow 0$ as $j \rightarrow \infty$. This completes the proof.

\section{Acknowledgements}

IM supported in part by Swiss National Science Foundation grant 200021137527. KR supported by the Swiss Government Excellence Scholarship.

The authors are grateful to the anonymous referees for very careful reading of this paper and suggesting a number of improvements.

\section{References}

Beghin, L., Orsingher, E., 2009. Fractional Poisson processes and related planar random motions. Electron. J. Probab. 14, 1790-1826.

Billingsley, P., 1968. Convergence of probability measures. John Wiley \& Sons, New York.

Bingham, N. H., 1971. Limit theorems for occupation times of Markov processes. Z. Wahrscheinlichkeitstheor. Verw. Geb. 17, 1-22.

Davydov, Y., Molchanov, I., Zuyev, S., 2008. Strictly stable distributions on convex cones. Electron. J. Probab. 13, 259-321.

Falconer, K., Liu, L., 2012. Multistable processes and localizability. Stoch. Models $28(3), 503-526$.

Falconer, K. J., Le Guével, R., Lévy Véhel, J., 2009. Localizable moving average symmetric stable and multistable processes. Stoch. Models 25 (4), 648-672. 
Falconer, K. J., Lévy Véhel, J., 2009. Multifractional, multistable, and other processes with prescribed local form. J. Theor. Probab. 22 (2), 375-401.

Fisz, M., Varadarajan, V. S., 1962/1963. A condition for absolute continuity of infinitely divisible distribution functions. Z. Wahrscheinlichkeitstheorie und Verw. Gebiete 1, 335-339.

Kingman, J. F. C., 1993. Poisson processes. Oxford: Clarendon Press.

Laskin, N., 2003. Fractional Poisson process. Commun. Nonlinear Sci. Numer. Simul. 8 (3-4), 201-213.

Le Guével, R., Lévy Véhel, J., 2012. A Ferguson-Klass-LePage series representation of multistable multifractional motions and related processes. Bernoulli 18 (4), 1099-1127.

Le Guével, R., Lévy Véhel, J., Liu, L., 2013. On two multistable extensions of stable Lévy motion and their semi-martingale representations. J. Theor. Probab.

URL http://dx.doi.org/10.1007/s10959-013-0528-6

Mainardi, F., Gorenflo, R., Scalas, E., 2004. A fractional generalization of the Poisson processes. Vietnam J. Math. 32, 53-64.

Meerschaert, M. M., Nane, E., Vellaisamy, P., 2011. The fractional Poisson process and the inverse stable subordinator. Electron. J. Probab. 16, 16001620 .

Meerschaert, M. M., Scheffler, H.-P., 2004. Limit theorems for continuous-time random walks with infinite mean waiting times. J. Appl. Prob. 41, 623-638.

Repin, O., Saichev, A., 2000. Fractional Poisson law. Radiophysics and Quantum Electronics 43 (9), 738-741.

Resnick, S. I., 1987. Extreme values, regular variation, and point processes. Applied Probability, Vol. 4, New York etc.: Springer-Verlag.

Seneta, E., 1976. Regularly varying functions. Lecture Notes in Mathematics. 508. Berlin-Heidelberg-New York: Springer-Verlag.

Uchaikin, V., Cahoy, D., Sibatov, R., 2008. Fractional processes: from Poisson to branching one. Int. J. Bifurcation Chaos Appl. Sci. Eng. 18 (9), 2717-2725.

Whitt, W., 2002. Stochastic-process limits. An introduction to stochasticprocess limits and their application to queues. New York, NY: Springer. 